\title{
EL-labelings and canonical spanning trees for subword complexes
}

\author{
Vincent Pilaud $\|^{\dagger}$ and Christian Stump ${ }^{2}$ \\ ${ }^{1}$ CNRS \& LIX, École Polytechnique, Palaiseau, France \\ ${ }^{2}$ Institut für Algebra, Zahlentheorie, Diskrete Mathematik, Universität Hannover, Germany
}

\begin{abstract}
We describe edge labelings of the increasing flip graph of a subword complex on a finite Coxeter group, and study applications thereof. On the one hand, we show that they provide canonical spanning trees of the facet-ridge graph of the subword complex, describe inductively these trees, and present their close relations to greedy facets. Searching these trees yields an efficient algorithm to generate all facets of the subword complex, which extends the greedy flip algorithm for pointed pseudotriangulations. On the other hand, when the increasing flip graph is a Hasse diagram, we show that the edge labeling is indeed an EL-labeling and derive further combinatorial properties of paths in the increasing flip graph. These results apply in particular to Cambrian lattices, in which case a similar EL-labeling was recently studied by M. Kallipoliti and H. Mühle.

Résumé. Nous décrivons des étiquetages d'arêtes du graphe des flips croissants d'un complexe de sous-mots sur un groupe de Coxeter fini, et nous en étudions certaines applications. D'une part, nous montrons qu'ils fournissent des arbres couvrants canoniques du graphe des flips du complexe de sous-mots, nous décrivons inductivement ces arbres, et nous présentons leurs liens étroits avec les facettes gloutonnes du complexe. Le parcours de ces arbres permet d'engendrer efficacement les facettes du complexe des sous-mots, généralisant ainsi l'algorithme de flips gloutons pour les pseudotriangulations. D'autre part, lorsque le graphe des flips croissants est un diagramme de Hasse, nous montrons que notre étiquetage d'arêtes est lexicographique et nous en déduisons des propriétés supplémentaires du graphe des flips croissants. Ces résultats s'appliquent en particulier aux treillis Cambriens pour lesquels un étiquetage lexicographique similaire a été récemment étudié par M. Kallipoliti and H. Mühle.
\end{abstract}

Keywords: EL-labelings, subword complexes, spanning trees, exhaustive generation, Möbius function.

Subword complexes on Coxeter groups were defined and studied by A. Knutson and E. Miller in the context of Gröbner geometry of Schubert varieties [KM04, KM05]. Type $A$ spherical subword complexes can be visually interpreted using pseudoline arrangements on primitive sorting networks. These were studied by V. Pilaud and M. Pocchiola [PP12] as combinatorial models for pointed pseudotriangulations of planar point sets [RSS08] and for multitriangulations of convex polygons [PS09]. These two families of geometric graphs extend in two different ways the family of triangulations of a convex polygon.

\footnotetext{
${ }^{\dagger}$ Partially supported by the spanish MICINN grant MTM2011-22792, by the French ANR grant EGOS 12 JS02 002 01, and by the European Research Project ExploreMaps (ERC StG 208471).

V.P. is grateful to M. Pocchiola for uncountable inspiring discussions on the subject. We thank M. Kallipoliti and H. Mühle for mentioning our construction in [KM12].
}

1365-8050 @ 2013 Discrete Mathematics and Theoretical Computer Science (DMTCS), Nancy, France 
The greedy flip algorithm was initially designed to generate all pointed pseudotriangulations of a given set of points or convex bodies in general position in the plane [PV96, BKPS06]. It was then extended in [PP12] to generate all pseudoline arrangements supported by a given primitive sorting network. The key step in this algorithm is to construct a spanning tree of the flip graph on the combinatorial objects, which has to be sufficiently canonical to be visited in polynomial time per node and polynomial working space.

In the present paper, we study natural edge lexicographic labelings of the increasing flip graph of a subword complex on any finite Coxeter group. As a first line of applications of these EL-labelings, we obtain canonical spanning trees of the flip graph of any subword complex. We provide alternative descriptions of these trees based on their close relations to greedy facets, which are defined and studied in this paper. Moreover, searching these trees provides an efficient algorithm to generate all facets of the subword complex. For type $A$ spherical subword complexes, the resulting algorithm is precisely that of [PP12], although the presentation is quite different.

The second line of applications of the EL-labelings concerns combinatorial properties ensuing from EL-shellability [Bjö80, BW96]. Indeed, when the increasing flip graph is the Hasse diagram of the increasing flip poset, this poset is EL-shellable, and we can compute its Möbius function. These results extend recent work of M. Kallipoliti and H. Mühle [KM12] on EL-shellability of N. Reading's Cambrian lattices [Rea06, Rea07], which are, for finite Coxeter groups, increasing flip posets of specific subword complexes studied by C. Ceballos, J.-P. Labbé and C. Stump [CLS13] and by the authors in [PS11].

This extended abstract presents the results and the main ideas of the paper [PS12]. We refer the reader to this paper for further details, examples, and all proofs which we omit here due to limited space.

\section{Edge labelings of graphs and posets}

In [Bjö80], A. Björner introduced EL-labelings of partially ordered sets to study topological properties of their order complexes. These labelings are edge labelings of the Hasse diagrams of the posets with certain combinatorial properties. In this paper, we consider edge labelings of finite, acyclic, directed graphs which might differ from the Hasse diagrams of their transitive closures.

\subsection{ER-labelings of graphs and associated spanning trees}

Let $G:=(V, E)$ be a finite, acyclic, directed graph. For $u, v \in V$, we write $u \rightarrow v$ if there is an edge from $u$ to $v$ in $G$, and $u \rightarrow v$ if there is a path $u=x_{1} \rightarrow x_{2} \rightarrow \cdots \rightarrow x_{\ell} \rightarrow x_{\ell+1}=v$ from $u$ to $v$ in $G$ (this path has length $\ell$ ). The interval $[u, v]$ in $G$ is the set of vertices $w \in V$ such that $u \rightarrow w \rightarrow v$.

An edge labeling of $G$ is a map $\lambda: E \rightarrow \mathbb{N}$. It induces a labeling of any path $p: x_{1} \rightarrow x_{2} \rightarrow \cdots \rightarrow x_{\ell} \rightarrow x_{\ell+1}$ given by $\lambda(p):=\lambda\left(x_{1} \rightarrow x_{2}\right) \cdots \lambda\left(x_{\ell} \rightarrow x_{\ell+1}\right)$. The path $p$ is $\lambda$-rising (resp. $\lambda$-falling) if $\lambda(p)$ is strictly increasing (resp. weakly decreasing). The labeling $\lambda$ is an edge rising labeling of $G$ (or ER-labeling for short) if there is a unique $\lambda$-rising path $p$ between any vertices $u, v \in V$ with $u-\rightarrow v$.

Remark 1.1 (Spanning trees) Let $u, v \in V$, and $\lambda: E \rightarrow \mathbb{N}$ be an ER-labeling of $G$. Then the union of all $\lambda$-rising paths from $u$ to any other vertex of the interval $[u, v]$ forms a spanning tree of $[u, v]$, rooted at and directed away from $u$. We call it the $\lambda$-source tree of $[u, v]$ and denote it by $\mathcal{T}_{\lambda}([u, v])$. Similarly, the union of all $\lambda$-rising paths from any vertex of the interval $[u, v]$ to $v$ forms a spanning tree of $[u, v]$, rooted at and directed towards $v$. We call it the $\lambda$-sink tree of $[u, v]$ and denote it by $\mathcal{T}_{\lambda}^{*}([u, v])$. In particular, if $G$ has a unique source and a unique sink, this provides two canonical spanning trees $\mathcal{T}_{\lambda}(G)$ and $\mathcal{T}_{\lambda}^{*}(G)$ for the graph $G$ itself. 
Example 1.2 (Cube) Consider the 1-skeleton $\square_{d}$ of the d-dimensional cube $[0,1]^{d}$, directed from vertex $\mathbf{0}:=(0, \ldots, 0)$ to vertex $\mathbf{1}:=(1, \ldots, 1)$. Its vertices are the elements of $\{0,1\}^{d}$ and its edges are the pairs of vertices which differ in a unique position. Note that $\varepsilon:=\left(\varepsilon_{1}, \ldots, \varepsilon_{d}\right)-\rightarrow \varepsilon^{\prime}:=\left(\varepsilon_{1}^{\prime}, \ldots, \varepsilon_{d}^{\prime}\right)$ if and only if $\varepsilon_{k} \leq \varepsilon_{k}^{\prime}$ for all $k \in[d]$.

For any edge $\varepsilon \rightarrow \varepsilon^{\prime}$ of $\square_{d}$, let $\lambda\left(\varepsilon \rightarrow \varepsilon^{\prime}\right)$ denote the unique position in $[d]$ where $\varepsilon$ and $\varepsilon^{\prime}$ differ. Then the map $\lambda$ is an ER-labeling of $\square_{d}$. If $\varepsilon \in\{0,1\}^{d} \backslash \mathbf{0}$, then the father of $\varepsilon$ in $\mathcal{T}_{\lambda}\left(\square_{d}\right)$ is obtained from $\varepsilon$ by changing its last 1 into a 0 . Similarly, if $\varepsilon \in\{0,1\}^{d} \backslash \mathbf{1}$, then the father of $\varepsilon$ in $\mathcal{T}_{\lambda}^{*}\left(\square_{d}\right)$ is obtained from $\varepsilon$ by changing its first 0 into a 1 . See Figure 1 .
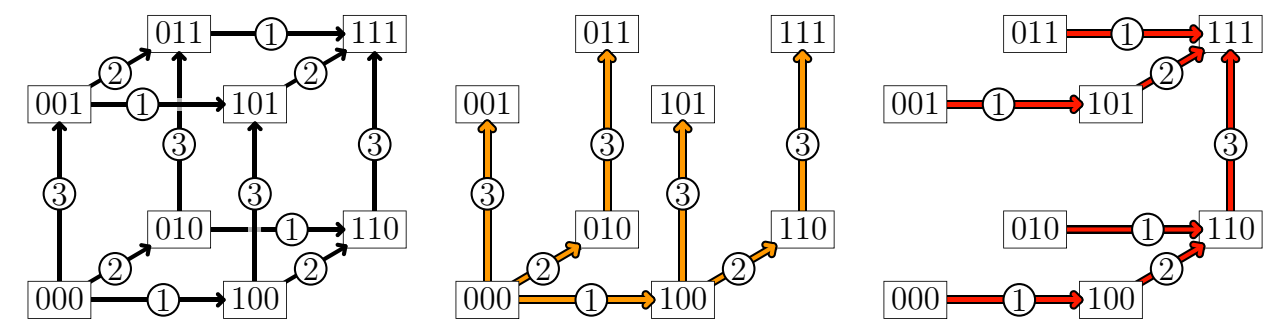

Fig. 1: An ER-labeling $\lambda$ of the 1-skeleton $\square_{3}$ of the 3 -cube, the $\lambda$-source tree $\mathcal{T}_{\lambda}\left(\square_{3}\right)$ and the $\lambda$-sink tree $\mathcal{T}_{\lambda}^{*}\left(\square_{3}\right)$.

\subsection{EL-labelings of graphs and posets}

Although ER-labelings of graphs are sufficient to produce canonical spanning trees, we need the following extension for further combinatorial properties. The labeling $\lambda: E \rightarrow \mathbb{N}$ is an edge lexicographic labeling of $G$ (or EL-labeling for short) if for any vertices $u, v \in V$ with $u-\rightarrow v$,

(i) there is a unique $\lambda$-rising path $p$ from $u$ to $v$, and

(ii) its labeling $\lambda(p)$ is lexicographically first among the labelings $\lambda\left(p^{\prime}\right)$ of all paths $p^{\prime}$ from $u$ to $v$.

For example, the ER-labeling of the 1-skeleton of the cube $\square_{d}$ presented in Example 1.2 is in fact an EL-labeling. Remember now that one can associate a finite poset to a finite acyclic directed graph and vice versa. Namely,

(i) the transitive closure of a finite acyclic directed graph $G=(V, E)$ is the finite poset $(V,-\rightarrow)$;

(ii) the Hasse diagram of a finite poset $P$ is the finite acyclic directed graph whose vertices are the elements of $P$ and whose edges are the cover relations in $P$, i.e. $u \rightarrow v$ if $u<_{P} v$ and there is no $w \in P$ such that $u<_{P} w<_{P} v$.

The transitive closure of the Hasse diagram of $P$ always coincides with $P$, but the Hasse diagram of the transitive closure of $G$ might as well be only a subgraph of $G$. An EL-labeling of the poset $P$ is an EL-labeling of the Hasse diagram of $P$. If such a labeling exists, then the poset is called EL-shellable.

As already mentioned, A. Björner [Bjö80] originally introduced EL-labelings of finite posets to study topological properties of their order complex. In particular, they provide a tool to compute the Möbius 
function of the poset. Recall that the Möbius function of the poset $P$ is the map $\mu: P \times P \rightarrow \mathbb{Z}$ defined recursively by

$$
\mu(u, v):= \begin{cases}1 & \text { if } u=v \\ -\sum_{u \leq_{P} w<_{P} v} \mu(u, w) & \text { if } u<_{P} v \\ 0 & \text { otherwise. }\end{cases}
$$

When the poset is EL-shellable, this function can be computed as follows.

Proposition 1.3 ([BW96, Proposition 5.7]) Let $\lambda$ be an EL-labeling of the poset P. For every $u, v \in P$ with $u \leq_{P} v$, we have

$$
\mu(u, v)=\operatorname{even}_{\lambda}(u, v)-\operatorname{odd}_{\lambda}(u, v),
$$

where $\operatorname{even}_{\lambda}(u, v)\left(\right.$ resp. odd $\left.{ }_{\lambda}(u, v)\right)$ denotes the number of even (resp. odd) length $\lambda$-falling paths from $u$ to $v$ in the Hasse diagram of $P$.

Example 1.4 (Cube) The directed 1-skeleton $\square_{d}$ of the d-dimensional cube $[0,1]^{d}$ is the Hasse diagram of the boolean poset. The edge labeling $\lambda$ of $\square_{d}$ of Example 1.2 is thus an EL-labeling of the boolean poset. Moreover, for any two vertices $\varepsilon \rightarrow \varepsilon^{\prime}$ of $\square_{d}$, there is a unique $\lambda$-falling path between $\varepsilon$ and $\varepsilon^{\prime}$, whose length is the Hamming distance $\delta\left(\varepsilon, \varepsilon^{\prime}\right):=\left|\left\{k \in[d] \mid \varepsilon_{k} \neq \varepsilon_{k}^{\prime}\right\}\right|$. The Möbius function is thus given by $\mu\left(\varepsilon, \varepsilon^{\prime}\right)=(-1)^{\delta\left(\varepsilon, \varepsilon^{\prime}\right)}$ if $\varepsilon \rightarrow \varepsilon^{\prime}$ and $\mu\left(\varepsilon, \varepsilon^{\prime}\right)=0$ otherwise. In particular, $\mu(\mathbf{0}, \mathbf{1})=(-1)^{d}$.

\section{Subword complexes on Coxeter groups}

\subsection{Subword complexes}

We consider a finite Coxeter system $(W, S)$, with root system $\Phi$ and simple roots $\left\{\alpha_{1}, \ldots, \alpha_{n}\right\}$. We fix a word $\mathrm{Q}:=\mathrm{q}_{1} \mathrm{q}_{2} \cdots \mathrm{q}_{m}$ on the generators of $S$, and an element $\rho \in W$. Background on Coxeter groups can be found in [Hum90].

A. Knutson and E. Miller [KM04] define the subword complex $\mathcal{S C}(\mathrm{Q}, \rho)$ to be the simplicial complex of those subwords of $\mathrm{Q}$ whose complements contain a reduced expression for $\rho$ as a subword. A vertex of $\mathcal{S C}(\mathrm{Q}, \rho)$ is a position of a letter in Q. We denote by $[m]:=\{1,2, \ldots, m\}$ the set of positions in Q. A facet of $\mathcal{S C}(\mathrm{Q}, \rho)$ is the complement of a set of positions which forms a reduced expression for $\rho$ in $\mathrm{Q}$. We denote by $\mathcal{F}(\mathrm{Q}, \rho)$ the set of facets of $\mathcal{S C}(\mathrm{Q}, \rho)$.

Example 2.1 Consider the type A Coxeter group $\mathfrak{S}_{4}$ generated by $\left\{\tau_{1}, \tau_{2}, \tau_{3}\right\}$, where $\tau_{i}:=(i \quad i+1)$. Consider $\mathrm{Q}^{\mathrm{ex}}:=\tau_{2} \tau_{3} \tau_{1} \tau_{3} \tau_{2} \tau_{1} \tau_{2} \tau_{3} \tau_{1}$ and $\rho^{\mathrm{ex}}:=[4,1,3,2]$. The reduced expressions of $\rho^{\mathrm{ex}}$ are $\tau_{2} \tau_{3} \tau_{2} \tau_{1}$, $\tau_{3} \tau_{2} \tau_{3} \tau_{1}$, and $\tau_{3} \tau_{2} \tau_{1} \tau_{3}$. Therefore, the facets of the subword complex $\mathcal{S C}\left(\mathrm{Q}^{\mathrm{ex}}, \rho^{\mathrm{ex}}\right)$ are $\{1,2,3,5,6\}$, $\{1,2,3,6,7\},\{1,2,3,7,9\},\{1,3,4,5,6\},\{1,3,4,6,7\},\{1,3,4,7,9\},\{2,3,5,6,8\},\{2,3,6,7,8\}$, $\{2,3,7,8,9\},\{3,4,5,6,8\},\{3,4,6,7,8\}$, and $\{3,4,7,8,9\}$. We will use this example throughout this paper to illustrate further notions.

Remark 2.2 There is a natural reversal operation on subword complexes. Namely,

$$
\mathcal{S C}\left(\mathrm{q}_{m} \cdots \mathrm{q}_{1}, \rho^{-1}\right)=\left\{\{m+1-i \mid i \in I\} \mid I \in \mathcal{S C}\left(\mathrm{q}_{1} \cdots \mathrm{q}_{m}, \rho\right)\right\} .
$$

We will use this operation to relate positive and negative labelings, facets and trees. 


\subsection{Inductive structure}

We denote by $\mathrm{Q}_{\vdash}:=\mathrm{q}_{2} \cdots \mathrm{q}_{m}$ and $\mathrm{Q}_{\dashv}:=\mathrm{q}_{1} \cdots \mathrm{q}_{m-1}$ the words on $S$ obtained from $\mathrm{Q}:=\mathrm{q}_{1} \cdots \mathrm{q}_{m}$ by deleting its first and last letters respectively. We denote by $\mathcal{X} \star z:=\{X \cup z \mid X \in \mathcal{X}\}$ the join of a collection $\mathcal{X}$ of subsets of $\mathbb{Z}$ with an element $z \in \mathbb{Z}$. We let $\ell(\rho)$ denote the length of $\rho \in W$ and we write $\rho \prec \mathrm{Q}$ when $\mathrm{Q}$ contains a reduced expression of $\rho$, i.e. when $\mathcal{S C}(\mathrm{Q}, \rho)$ is non-empty.

We can decompose inductively the facets of the subword complex $\mathcal{S C}(\mathrm{Q}, \rho)$ according on whether or not they contain the last letter of Q. Denoting by $\varepsilon$ the empty word and by $e$ the identity of $W$, we have $\mathcal{F}(\varepsilon, e)=\{\emptyset\}$ and $\mathcal{F}(\varepsilon, \rho)=\emptyset$ if $\rho \neq e$. For a non-empty word $\mathrm{Q}$ on $S$, the set $\mathcal{F}(\mathrm{Q}, \rho)$ is given by

(i) $\mathcal{F}\left(\mathrm{Q}_{\dashv}, \rho q_{m}\right)$ if $m$ appears in none of the facets of $\mathcal{S C}(\mathrm{Q}, \rho)$ (equivalently if $\rho \nprec \mathrm{Q}_{\dashv}$ );

(ii) $\mathcal{F}\left(\mathrm{Q}_{\dashv}, \rho\right) \star m$ if $m$ appears in all the facets of $\mathcal{S C}(\mathrm{Q}, \rho)$ (equivalently if $\ell\left(\rho q_{m}\right)>\ell(\rho)$ );

(iii) $\mathcal{F}\left(\mathrm{Q}_{\dashv}, \rho q_{m}\right) \sqcup\left(\mathcal{F}\left(\mathrm{Q}_{\dashv}, \rho\right) \star m\right)$ otherwise.

By reversal (see Remark 2.2, there is also a similar inductive decomposition of the facets of the subword complex $\mathcal{S C}(\mathrm{Q}, \rho)$ according on whether or not they contain the first letter of Q. Although we will only use these decompositions for the facets $\mathcal{F}(\mathrm{Q}, \rho)$, they extend to the whole subword complex $\mathcal{S C}(\mathrm{Q}, \rho)$ and are used to obtain the following result.

Theorem 2.3 ([KM04, Corollary 3.8]) The subword complex $\mathcal{S C}(\mathrm{Q}, \rho)$ is either a simplicial sphere or a simplicial ball.

\subsection{Flips and roots}

Let $I$ be a facet of $\mathcal{S C}(\mathrm{Q}, \rho)$ and $i$ be a position in $I$. If there exists a facet $J$ of $\mathcal{S C}(\mathrm{Q}, \rho)$ and a position $j \in J$ such that $I \backslash i=J \backslash j$, we say that $I$ and $J$ are adjacent facets, that $i$ is fippable in $I$, and that $J$ is obtained from $I$ by flipping $i$. Note that, if $i$ is flippable, then $J$ and $j$ are unique by Theorem 2.3. We denote by $\mathcal{G}(\mathrm{Q}, \rho)$ the graph of flips, whose vertices are the facets of $\mathcal{S C}(\mathrm{Q}, \rho)$ and whose edges are pairs of adjacent facets. That is, $\mathcal{G}(\mathrm{Q}, \rho)$ is the ridge graph of the simplicial complex $\mathcal{S C}(\mathrm{Q}, \rho)$. This graph is connected by Theorem 2.3 It can moreover be naturally oriented by the direction of the flips as follows. Let $I$ and $J$ be two adjacent facets of $\mathcal{S C}(\mathrm{Q}, \rho)$ with $I \backslash i=J \backslash j$. We say that the flip from $I$ to $J$ is increasing if $i<j$. We then orient the corresponding edge of $\mathcal{G}(\mathrm{Q}, \rho)$ from $I$ to $J$.

Example 2.4 Figure 2 shows the increasing flip graph $\mathcal{G}\left(\mathrm{Q}^{\mathrm{ex}}, \rho^{\mathrm{ex}}\right)$ for the subword complex $\mathcal{S C}\left(\mathrm{Q}^{\mathrm{ex}}, \rho^{\mathrm{ex}}\right)$ of Example 2.1. The facets of $\mathcal{S C}\left(\mathrm{Q}^{\mathrm{ex}}, \rho^{\mathrm{ex}}\right)$ appear in lexicographic order from left to right.

Remark 2.5 The increasing fip graph of $\mathcal{S C}(\mathrm{Q}, \rho)$ was already considered by A. Knutson and E. Miller [KM04 Remark 4.5]. It carries various combinatorial informations about the subword complex $\mathcal{S C}(\mathrm{Q}, \rho)$. In particular, since the lexicographic order on the facets of $\mathcal{S C}(\mathrm{Q}, \rho)$ is a shelling order for $\mathcal{S C}(\mathrm{Q}, \rho)$, the $h$-vector of the subword complex $\mathcal{S C}(\mathrm{Q}, \rho)$ is the in-degree sequence of the increasing flip graph $\mathcal{G}(\mathrm{Q}, \rho)$.

We consider flips as elementary operations on subword complexes. In practice, the necessary information to perform flips in a facet $I$ of $\mathcal{S C}(\mathrm{Q}, \rho)$ is encoded in its root function $\mathrm{r}(I, \cdot):[m] \rightarrow \Phi$ defined by

$$
\mathrm{r}(I, k):=\Pi \mathrm{Q}_{[k-1] \backslash I}\left(\alpha_{q_{k}}\right),
$$

where $\Pi \mathrm{Q}_{X}$ denotes the product of the reflections $q_{x} \in \mathrm{Q}$ for $x \in X$. The root function was introduced by C. Ceballos, J.-P. Labbé and C. Stump [CLS13] and its main properties can be found in [CLS13, Lemmas 
3.3 and 3.6]. Essentially, an element $i$ of a facet $I$ is flippable if and only if $r(I, i) \in\{ \pm \beta \mid \beta \in \operatorname{inv}(\rho)\}$, and then $i$ flips to the unique position $j \notin I$ such that $\mathrm{r}(I, j) \in\{ \pm \mathrm{r}(I, i)\}$. Moreover, $\mathrm{r}(I, i)=\mathrm{r}(I, j) \in \Phi^{+}$ if $i<j$ (increasing flip), while $\mathrm{r}(I, i)=-\mathrm{r}(I, j) \in \Phi^{-}$if $i>j$ (decreasing flip). The root configuration of the facet $I$ is the multiset $\mathrm{R}(I):=\{\{\mathrm{r}(I, i) \mid i$ flippable in $I\}$. We extensively studied root configurations in [PS11] in the construction of brick polytopes for spherical subword complexes.

\section{EL-labelings and spanning trees for the subword complex}

\subsection{EL-labelings of the increasing flip graph}

We now define two natural edge labelings of the increasing flip graph $\mathcal{G}(\mathrm{Q}, \rho)$. Let $I$ and $J$ be two adjacent facets of $\mathcal{S C}(\mathrm{Q}, \rho)$, with $I \backslash i=J \backslash j$ and $i<j$. We label the edge $I \rightarrow J$ of $\mathcal{G}(\mathrm{Q}, \rho)$ with the positive edge label $\mathrm{p}(I \rightarrow J):=i$ and with the negative edge label $\mathrm{n}(I \rightarrow J):=j$. We call $\mathrm{p}: E(\mathcal{G}(\mathrm{Q}, \rho)) \rightarrow[m]$ the positive edge labeling and $\mathrm{n}: E(\mathcal{G}(\mathrm{Q}, \rho)) \rightarrow[m]$ the negative edge labeling of the increasing flip graph $\mathcal{G}(\mathrm{Q}, \rho)$. The terms "positive" and "negative" emphasize the fact that the roots $\mathrm{r}(I, \mathrm{p}(I \rightarrow J))$ and $\mathrm{r}(J, \mathrm{n}(I \rightarrow J))$ are always positive and negative roots respectively. The positive and negative edge labelings are clearly reverse to one another (see Remark 2.2.

Example 3.1 Consider the subword complex $\mathcal{S C}\left(\mathrm{Q}^{\mathrm{ex}}, \rho^{\mathrm{ex}}\right)$ of Example 2.1 We have represented on Figure 2 the positive and negative edge labelings $\mathrm{p}$ and $\mathrm{n}$. Since we have represented the graph $\mathcal{G}\left(\mathrm{Q}^{\mathrm{ex}}, \rho^{\mathrm{ex}}\right)$ such that the flips are increasing from left to right, each edge has its positive label on the left and its negative label on the right.

Our main result concerns the positive and negative edge labelings of the increasing flip graph.

Theorem 3.2 The positive edge labeling $\mathrm{p}$ and the negative edge labeling $\mathrm{n}$ are both EL-labelings of the increasing flip graph.

For Cambrian lattices, whose Hasse diagrams were shown to be particular cases of increasing flip graphs in [PS11, Section 6], a similar result was recently obtained by M. Kallipoliti and H. Mühle in [KM12].

In Sections 3.2 and 3.3, we present applications of Theorem 3.2 to the construction of canonical spanning trees and to the generation of the facets of the subword complex. Further combinatorial applications of this theorem are also discussed in Section 4

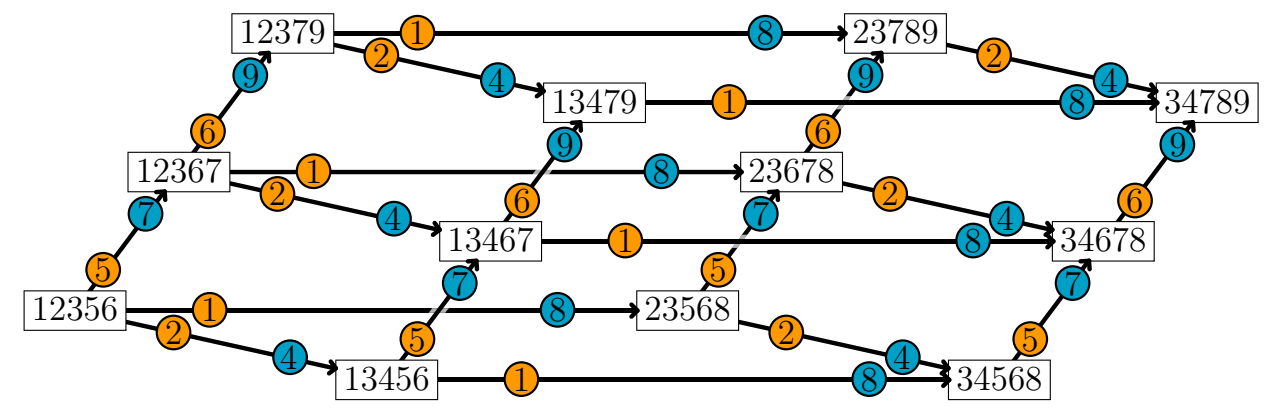

Fig. 2: The positive and negative edge labelings $\mathrm{p}$ and $\mathrm{n}$ of $\mathcal{G}\left(\mathrm{Q}^{\mathrm{ex}}, \rho^{\mathrm{ex}}\right)$. Each edge has its positive label on the left (orange) and its negative label on the right (blue). 
Before going on, we want to give a very brief idea of the proof of Theorem 3.2 We refer the interested reader to the complete proof in [PS12]. To prove the existence of a p-rising path between any two comparable facets of $\mathcal{S C}(\mathrm{Q}, \rho)$, we use a procedure which improves locally a path by restriction of $\mathcal{S C}(\mathrm{Q}, \rho)$ to a dihedral parabolic subgroup. The uniqueness and lexicographic property of the p-rising are then obtained from the following proposition.

Proposition 3.3 Let $I_{1} \rightarrow \cdots \rightarrow I_{\ell+1}$ be a path of increasing flips, and define $\mathrm{p}_{k}:=\mathrm{p}\left(I_{k} \rightarrow I_{k+1}\right)$ and $\mathrm{n}_{k}:=\mathrm{n}\left(I_{k} \rightarrow I_{k+1}\right)$. Then, for all $k \in[\ell]$, we have

$$
\min \left\{\mathrm{p}_{k}, \ldots, \mathrm{p}_{\ell}\right\}=\min \left(I_{k} \backslash I_{\ell+1}\right) \quad \text { and } \max \left\{\mathrm{n}_{1}, \ldots, \mathrm{n}_{k}\right\}=\max \left(I_{k+1} \backslash I_{1}\right)
$$

Moreover, the path is $\mathrm{p}$-rising if and only if $\mathrm{p}_{k}=\min \left(I_{k} \backslash I_{\ell+1}\right)$ for all $k \in[\ell]$, while the path is $\mathrm{n}$-rising if and only if $\mathrm{n}_{k}=\max \left(I_{k+1} \backslash I_{1}\right)$ for all $k \in[\ell]$.

\subsection{Greedy facets}

We now characterize the unique source and sink of the increasing flip graph $\mathcal{G}(\mathrm{Q}, \rho)$.

Proposition 3.4 The lexicographically smallest (resp. largest) facet of $\mathcal{S C}(\mathrm{Q}, \rho)$ is the unique source (resp. sink) of $\mathcal{G}(\mathrm{Q}, \rho)$.

We call positive (resp. negative) greedy facet and denote by $\mathrm{P}(\mathrm{Q}, \rho)$ (resp. $\mathrm{N}(\mathrm{Q}, \rho)$ ) the unique source (resp. sink) of the graph $\mathcal{G}(\mathrm{Q}, \rho)$ of increasing flips. The term "positive" (resp. "negative") emphasizes the fact that $\mathrm{P}(\mathrm{Q}, \rho)$ (resp. $\mathrm{N}(\mathrm{Q}, \rho)$ ) is the unique facet of $\mathcal{S C}(\mathrm{Q}, \rho)$ whose root configuration is a subset of positive (resp. negative) roots, while the term "greedy" refers to the greedy properties of these facets. The greedy facets $\mathrm{P}(\mathrm{Q}, \rho)$ and $\mathrm{N}(\mathrm{Q}, \rho)$ are reverse to one another (see Remark 2.2). Namely, $\mathrm{N}\left(\mathrm{q}_{m} \cdots \mathrm{q}_{1}, \rho^{-1}\right)=\left\{m+1-p \mid p \in \mathrm{P}\left(\mathrm{q}_{1} \cdots \mathrm{q}_{m}, \rho\right)\right\}$.

Example 3.5 The positive and negative greedy facets of the subword complex $\mathcal{S C}\left(\mathrm{Q}^{\mathrm{ex}}, \rho^{\mathrm{ex}}\right)$ presented in Example 2.1 are respectively $\mathrm{P}\left(\mathrm{Q}^{\mathrm{ex}}, \rho^{\mathrm{ex}}\right)=\{1,2,3,5,6\}$ and $\mathrm{N}\left(\mathrm{Q}^{\mathrm{ex}}, \rho^{\mathrm{ex}}\right)=\{3,4,7,8,9\}$. They appear respectively as the leftmost and rightmost facets in Figure 2 .

We have seen in Theorem 3.2 that for any two facets $I, J \in \mathcal{F}(\mathrm{Q}, \rho)$ such that $I-\rightarrow J$, there is a p-rising (resp. n-rising) path from $I$ to $J$. In particular, there is always a p-rising (resp. n-rising) path from $\mathrm{P}(\mathrm{Q}, \rho)$ to $\mathrm{N}(\mathrm{Q}, \rho)$. It turns out that there is also at least one $\mathrm{p}$-falling (resp. $\mathrm{n}$-falling) path from $\mathrm{P}(\mathrm{Q}, \rho)$ to $\mathrm{N}(\mathrm{Q}, \rho)$ if the subword complex $\mathcal{S C}(\mathrm{Q}, \rho)$ is spherical.

Proposition 3.6 For any spherical subword complex $\mathcal{S C}(\mathrm{Q}, \rho)$, there is always a $\mathrm{p}$-falling and an $\mathrm{n}$ falling path from $\mathrm{P}(\mathrm{Q}, \rho)$ to $\mathrm{N}(\mathrm{Q}, \rho)$.

Note that this proposition fails if we drop the condition that $\mathcal{S C}(\mathrm{Q}, \rho)$ is spherical, as illustrated in the subword complex $\mathcal{S C}\left(\mathrm{Q}^{\mathrm{ex}}, \rho^{\mathrm{ex}}\right)$ of Example 2.1.

\subsection{Spanning trees}

As discussed in Remark 1.1, the edge labelings $\mathrm{p}$ and $\mathrm{n}$ automatically produce canonical spanning trees of any interval of the increasing flip graph $\mathcal{G}(\mathrm{Q}, \rho)$. Since $\mathcal{G}(\mathrm{Q}, \rho)$ has a unique source $\mathrm{P}(\mathrm{Q}, \rho)$ and a unique sink $\mathrm{N}(\mathrm{Q}, \rho)$, we obtain in particular four spanning trees of the graph $\mathcal{G}(\mathrm{Q}, \rho)$ itself. The goal of this section is to give alternative descriptions of these four spanning trees. 
We call respectively positive source tree, positive sink tree, negative source tree, and negative sink tree, and denote respectively by $\mathcal{P}(\mathrm{Q}, \rho), \mathcal{P}^{*}(\mathrm{Q}, \rho), \mathcal{N}(\mathrm{Q}, \rho)$, and $\mathcal{N}^{*}(\mathrm{Q}, \rho)$, the p-source, p-sink, n-source, and $\mathrm{n}$-sink trees of $\mathcal{G}(\mathrm{Q}, \rho)$. The tree $\mathcal{P}(\mathrm{Q}, \rho)$ (resp. $\mathcal{N}(\mathrm{Q}, \rho)$ ) is formed by all p-rising (resp. n-rising) paths from the positive greedy facet $\mathrm{P}(\mathrm{Q}, \rho)$ to all the facets of $\mathcal{S C}(\mathrm{Q}, \rho)$. Both $\mathcal{P}(\mathrm{Q}, \rho)$ and $\mathcal{N}(\mathrm{Q}, \rho)$ are rooted at and directed away from the positive greedy facet $\mathrm{P}(\mathrm{Q}, \rho)$. The tree $\mathcal{P}^{*}(\mathrm{Q}, \rho)$ (resp. $\mathcal{N}^{*}(\mathrm{Q}, \rho)$ ) is formed by all p-rising (resp. n-rising) paths from all the facets of $\mathcal{S C}(\mathrm{Q}, \rho)$ to the negative greedy facet $\mathrm{N}(\mathrm{Q}, \rho)$. Both $\mathcal{P}^{*}(\mathrm{Q}, \rho)$ and $\mathcal{N}^{*}(\mathrm{Q}, \rho)$ are rooted at and directed towards the negative greedy facet $\mathrm{N}(\mathrm{Q}, \rho)$. Note that the positive source and negative sink trees (resp. the positive sink and the negative source trees) are reverse to one another (see Remark 2.2.

Example 3.7 Consider the subword complex $\mathcal{S C}\left(\mathrm{Q}^{\mathrm{ex}}, \rho^{\mathrm{ex}}\right)$ of Example 2.1 Figure 3 represents the trees $\mathcal{P}\left(\mathrm{Q}^{\mathrm{ex}}, \rho^{\mathrm{ex}}\right), \mathcal{P}^{*}\left(\mathrm{Q}^{\mathrm{ex}}, \rho^{\mathrm{ex}}\right), \mathcal{N}\left(\mathrm{Q}^{\mathrm{ex}}, \rho^{\mathrm{ex}}\right)$, and $\mathcal{N}^{*}\left(\mathrm{Q}^{\mathrm{ex}}, \rho^{\mathrm{ex}}\right)$. Observe that these four canonical spanning trees of $\mathcal{G}(\mathrm{Q}, \rho)$ are all different in general.

We now give a direct description of the father of a facet $I$ in $\mathcal{P}^{*}(\mathrm{Q}, \rho)$ and $\mathcal{N}(\mathrm{Q}, \rho)$.

Proposition 3.8 Let I be a facet of $\mathcal{S C}(\mathrm{Q}, \rho)$. If $I \neq \mathrm{N}(\mathrm{Q}, \rho)$, then the father of I in $\mathcal{P}^{*}(\mathrm{Q}, \rho)$ is obtained from I by flipping the smallest position in $I \backslash \mathrm{N}(\mathrm{Q}, \rho)$. Similarly, if $I \neq \mathrm{P}(\mathrm{Q}, \rho)$, then the father of $I$ in $\mathcal{N}(\mathrm{Q}, \rho)$ is obtained from I by flipping the largest position in $I \backslash \mathrm{P}(\mathrm{Q}, \rho)$.

We now focus on the positive source tree $\mathcal{P}(\mathrm{Q}, \rho)$ and on the negative sink tree $\mathcal{N}^{*}(\mathrm{Q}, \rho)$, and provide two different descriptions of them. The first is an inductive description of $\mathcal{P}(\mathrm{Q}, \rho)$ and $\mathcal{N}^{*}(\mathrm{Q}, \rho)$ (see Proposition 3.10p. The second is a direct description of the father of a facet $I$ in $\mathcal{P}(\mathrm{Q}, \rho)$ and $\mathcal{N}^{*}(\mathrm{Q}, \rho)$ in terms of greedy prefixes and suffixes of $I$ (see Proposition 3.11). These descriptions mainly rely on the following property of the greedy facets.

Proposition 3.9 If $m$ is a flippable position of $\mathrm{N}(\mathrm{Q}, \rho)$, then $\mathrm{N}\left(\mathrm{Q}_{\dashv}, \rho q_{m}\right)$ is obtained from $\mathrm{N}(\mathrm{Q}, \rho)$ by flipping $m$. Similarly, if 1 is a flippable position of $\mathrm{P}(\mathrm{Q}, \rho)$, then $\mathrm{P}\left(\mathrm{Q}_{\vdash}, q_{1} \rho\right)$ is obtained from $\mathrm{P}(\mathrm{Q}, \rho)$ by flipping 1 and shifting to the left.

Using Proposition 3.9, we can describe inductively the two trees $\mathcal{P}(\mathrm{Q}, \rho)$ and $\mathcal{N}^{*}(\mathrm{Q}, \rho)$. The induction follows the induction formulas for the facets $\mathcal{F}(\mathrm{Q}, \rho)$ presented in Section 2.2 Remember that we denote the deletion of the first or last letter in $Q:=q_{1} \cdots q_{m}$ by $Q_{\vdash}:=q_{2} \cdots q_{m}$ and $Q_{\dashv}:=q_{1} \cdots q_{m-1}$ respectively. For a tree $\mathcal{T}$ whose vertices are subsets of $\mathbb{Z}$ and for an element $z \in \mathbb{Z}$, we denote by $\mathcal{T} \star z$ the tree with a vertex $X \cup z$ for each vertex $X$ of $\mathcal{T}$ and an edge $X \cup z \rightarrow Y \cup z$ for each edge $X \rightarrow Y$ of $\mathcal{T}$.

The inductive description of the negative sink tree $\mathcal{N}^{*}(\mathrm{Q}, \rho)$ is based on the right induction formula. For the empty word $\varepsilon$, the tree $\mathcal{N}^{*}(\varepsilon, e)$ is formed by the unique facet $\emptyset$ of $\mathcal{S C}(\varepsilon, e)$, and the tree $\mathcal{N}^{*}(\varepsilon, \rho)$ is empty if $\rho \neq e$. Otherwise, $\mathcal{N}^{*}(\mathrm{Q}, \rho)$ is obtained as follows.

Proposition 3.10 For a non-empty word $\mathrm{Q}$, the tree $\mathcal{N}^{*}(\mathrm{Q}, \rho)$ equals

(i) $\mathcal{N}^{*}\left(\mathrm{Q}_{\dashv}, \rho q_{m}\right)$ if $m$ appears in none of the facets of $\mathcal{S C}(\mathrm{Q}, \rho)$;

(ii) $\mathcal{N}^{*}\left(\mathrm{Q}_{\dashv}, \rho\right) \star m$ if $m$ appears in all the facets of $\mathcal{S C}(\mathrm{Q}, \rho)$;

(iii) the disjoint union of $\mathcal{N}^{*}\left(\mathrm{Q}_{\dashv}, \rho q_{m}\right)$ and $\mathcal{N}^{*}\left(\mathrm{Q}_{\dashv}, \rho\right) \star m$, with an additional edge from $\mathrm{N}\left(\mathrm{Q}_{\dashv}, \rho q_{m}\right)$ to $\mathrm{N}(\mathrm{Q}, \rho)=\mathrm{N}\left(\mathrm{Q}_{\dashv}, \rho\right) \cup m$, otherwise. 

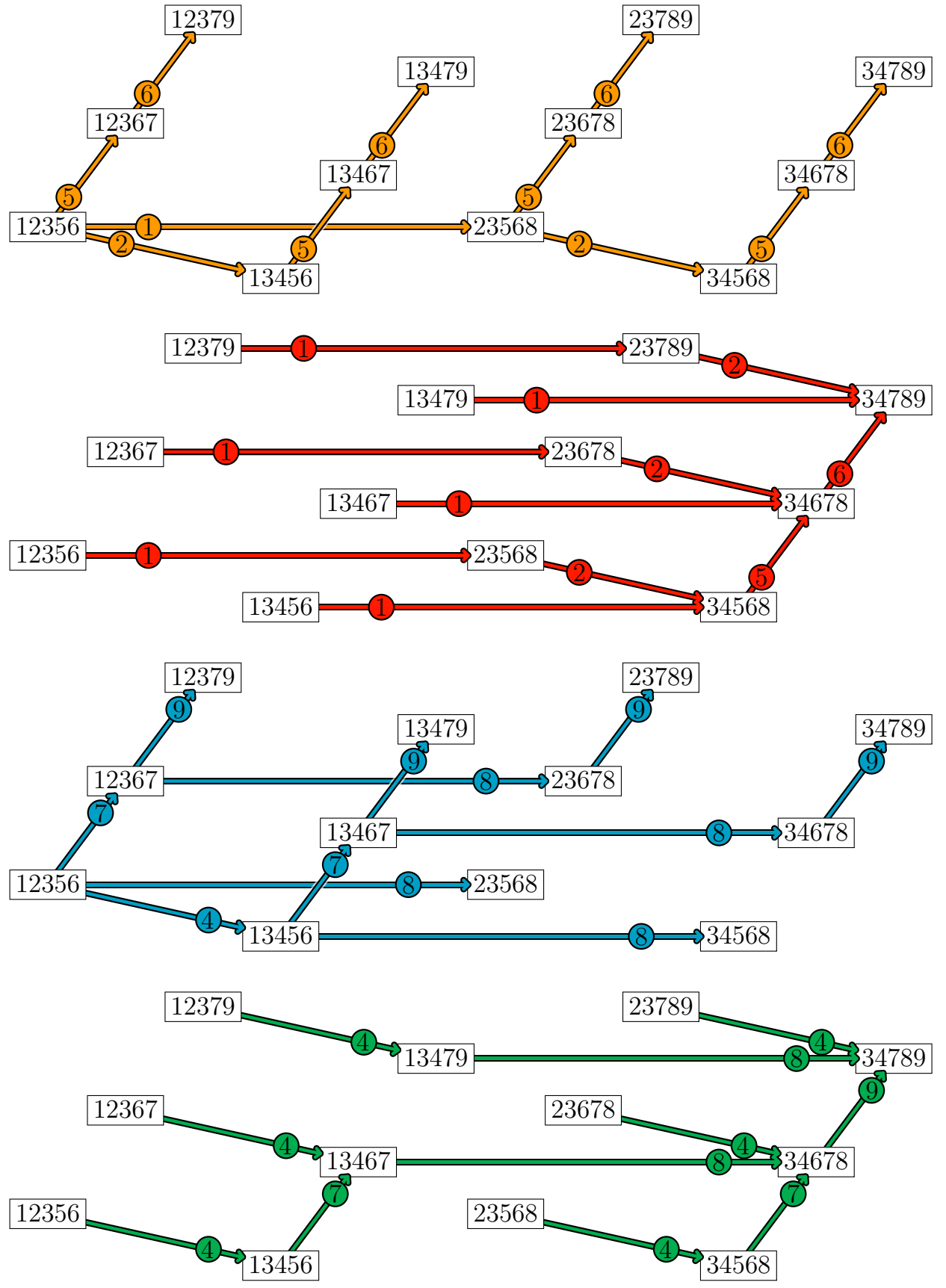

Fig. 3: The positive source tree $\mathcal{P}\left(\mathrm{Q}^{\mathrm{ex}}, \rho^{\mathrm{ex}}\right)$, the positive sink tree $\mathcal{P}^{*}\left(\mathrm{Q}^{\mathrm{ex}}, \rho^{\mathrm{ex}}\right)$, the negative source tree $\mathcal{N}\left(\mathrm{Q}^{\text {ex }}, \rho^{\text {ex }}\right)$, and the negative sink tree $\mathcal{N}^{*}\left(\mathrm{Q}^{\text {ex }}, \rho^{\text {ex }}\right)$ of the subword complex $\mathcal{S C}\left(\mathrm{Q}^{\text {ex }}, \rho^{\text {ex }}\right)$ of Example 2.1 
A similar inductive description of the positive source tree $\mathcal{P}(\mathrm{Q}, \rho)$ can be obtained from the left induction formula. See [PS12].

We now give a direct characterization of the father of a facet $I$ of $\mathcal{S C}(\mathrm{Q}, \rho)$ in the positive source and negative sink trees $\mathcal{P}(\mathrm{Q}, \rho)$ and $\mathcal{N}^{*}(\mathrm{Q}, \rho)$. This description can be understood in terms of the longest greedy prefix or suffix of $I$.

Proposition 3.11 Let I be a facet of $\mathcal{S C}(\mathrm{Q}, \rho)$. If $I \neq N(\mathrm{Q}, \rho)$, then the father of I in $\mathcal{N}^{*}(\mathrm{Q}, \rho)$ is obtained from $I$ by flipping the smallest position $x \in[m]$ such that $I \cap[x] \neq \mathrm{N}\left(\mathrm{q}_{1} \cdots \mathrm{q}_{x}, \Pi \mathrm{Q}_{[x] \backslash I}\right)$. Similarly, if $I \neq \mathrm{P}(\mathrm{Q}, \rho)$, then the father of $I$ in $\mathcal{P}(\mathrm{Q}, \rho)$ is obtained from I by flipping the largest position $x \in[m]$ such that $\{i-x \mid i \in I \backslash[x]\} \neq \mathrm{P}\left(\mathrm{q}_{x+1} \cdots \mathrm{q}_{m}, \Pi \mathrm{Q}_{[x+1, m] \backslash I}\right)$.

\subsection{Greedy flip algorithm}

The initial motivation of this paper was to find efficient algorithms for the exhaustive generation of the set $\mathcal{F}(\mathrm{Q}, \rho)$ of facets of the subword complex $\mathcal{S C}(\mathrm{Q}, \rho)$. The properties of the subword complex described in Sections 2.2 and 2.3 already provide two immediate enumeration algorithms. First, the inductive structure of $\mathcal{F}(\mathrm{Q}, \rho)$ yields an inductive algorithm whose running time per facet is polynomial. The second option is an exploration of the flip graph $\mathcal{G}(\mathrm{Q}, \rho)$, whose running time is still polynomial per facet. The problem of a naive exploration is that we would need to store all facets of $\mathcal{F}(\mathrm{Q}, \rho)$ during the algorithm, which may require an exponential working space. Using the canonical spanning trees constructed in this paper, we can bypass this difficulty: we avoid to store all visited facets while preserving the same running time. The greedy flip algorithm generates all facets of the subword complex $\mathcal{S C}(\mathrm{Q}, \rho)$ by a depth first search procedure on one of the four canonical spanning trees described in Section 3.3 The preorder traversal of the tree also provides an iterator on the facets of $\mathcal{S C}(\mathrm{Q}, \rho)$. We refer to [PS12] for a discussion on the complexity and on an implementation of this algorithm. This algorithm is similar to that of [BKPS06] for pointed triangulations and that of [BP12] for primitive sorting networks.

\section{Further combinatorial properties of the EL-labelings}

In this section, we discuss some implications of the EL-labelings of the increasing flip graph presented in Section 3.1 These results concern combinatorial properties of the increasing flip poset $\Gamma(\mathrm{Q}, \rho)$, defined as the transitive closure of the increasing flip graph $\mathcal{G}(\mathrm{Q}, \rho)$. The key property for the validity of these results is that the increasing flip graph $\mathcal{G}(\mathrm{Q}, \rho)$ coincides with the Hasse diagram of the increasing flip poset $\Gamma(\mathrm{Q}, \rho)$ (see the discussion in the beginning of Section 1.2). We first characterize and study the subword complexes which fulfill this property.

We say that the subword complex $\mathcal{S C}(\mathrm{Q}, \rho)$ has a double root if there is a facet $I \in \mathcal{S C}(\mathrm{Q}, \rho)$ and two distinct positions $i \neq j \in[m]$ both flippable in $I$ such that $\mathrm{r}(I, i)=\mathrm{r}(I, j)$. Otherwise, we say that the subword complex $\mathcal{S C}(\mathrm{Q}, \rho)$ is double root free. We focus on double root free subword complexes due to the following characterization.

Proposition 4.1 The subword complex $\mathcal{S C}(\mathrm{Q}, \rho)$ is double root free if and only if its increasing flip graph $\mathcal{G}(\mathrm{Q}, \rho)$ coincides with the Hasse diagram of its increasing flip poset $\Gamma(\mathrm{Q}, \rho)$.

Intervals in the increasing flip graph of a double root free subword complex have the following property.

Proposition 4.2 Let $I$ and $J$ be two facets of a double root free subword complex $\mathcal{S C}(\mathrm{Q}, \rho)$. Then the intersection $I \cap J$ is contained in all facets of the interval $[I, J]$ in the increasing flip graph $\mathcal{G}(\mathrm{Q}, \rho)$. 
Corollary 4.3 There is at most one $\mathrm{p}$-falling (resp. $\mathrm{n}$-falling) path between any two facets $I$ and $J$ of a double root free subword complex $\mathcal{S C}(\mathrm{Q}, \rho)$. If it exists, its length is given by $|I \backslash J|=|J \backslash I|$.

Corollary 4.4 Let $I$ and $J$ be two facets of a double root free subword complex such that $I-\rightarrow J$. The unique p-rising (resp. $\mathrm{n}$-rising) path from I to J has maximal length among all path from I to J. Moreover, if there is a p-falling (resp. $\mathrm{n}$-falling) path from I to $J$, it has minimal length.

Remark 4.5 Note that the conclusions of Proposition 4.2, Corollary 4.3, and Corollary 4.4 do indeed not hold if $\mathcal{S C}(\mathrm{Q}, \rho)$ has double roots. This situation reduces to the situation of type $A_{1}$ with generator $s$ for the word $\mathrm{Q}=\mathrm{sss}$ and the element $\rho=s$, which contradicts the three statements.

Corollary 4.6 The Möbius function on the increasing flip poset $\Gamma(\mathrm{Q}, \rho)$ of a double root free subword complex $\mathcal{S C}(\mathrm{Q}, \rho)$ is given by

$$
\mu(I, J)= \begin{cases}(-1)^{|J \backslash I|} & \text { if there is a p-falling (resp. n-falling) path from } I \text { to } J \\ 0 & \text { otherwise. }\end{cases}
$$

By this corollary, we can compute the Möbius function of an interval $[I, J]$ of the increasing flip poset as soon as we can decide whether or not there is a p-falling path from $I$ to $J$. According to Proposition 3.6 . there is always a p-falling path from the positive greedy facet to the negative greedy facet of a spherical subword complex. We therefore obtain the following statement.

Corollary 4.7 In a spherical double root free subword complex $\mathcal{S C}(\mathrm{Q}, \rho)$, we have

$$
\mu(\mathrm{P}(\mathrm{Q}, \rho), \mathrm{N}(\mathrm{Q}, \rho))=(-1)^{|\mathrm{Q}|-\ell(\rho)} .
$$

Observe again that this result fails if we drop the condition that $\mathcal{S C}(\mathrm{Q}, \rho)$ is spherical. The subword complex $\mathcal{S C}\left(\mathrm{Q}^{\mathrm{ex}}, \rho^{\mathrm{ex}}\right)$ of Example 2.1 provides a counter-example.

Example 4.8 (Cambrian lattices) We finally want to recall that cluster complexes of finite types are particular examples of subword complexes, see [CLS13]. This implies that Cambrian lattices of finite types are indeed increasing flip graphs, see [PS11]. Our construction thus proves that Cambrian lattices of finite types are EL-shellable. This result was as well obtained by M. Kallipoliti and H. Mühle in [KM12]. We want to emphasize that the two resulting labelings differ, as do the two resulting spanning trees. We refer to the long version of this paper [PS12] for further details.

Example 4.9 (Duplicated words) Fix an element $\rho \in W$ and a reduced expression of it. Consider $a$ word $\mathrm{Q}^{\mathrm{dup}}$ obtained by duplicating $d \leq \ell(\rho)$ letters in this reduced expression. Any facet of the subword complex $\mathcal{S C}\left(\mathrm{Q}^{\text {dup }}, \rho\right)$ contains precisely one position among each pair of duplicated letters, and no other position. Therefore, the subword complex $\mathcal{S C}\left(\mathrm{Q}^{\mathrm{dup}}, \rho\right)$ is the boundary complex of a $d$ dimensional cross-polytope, its increasing flip graph $\mathcal{G}\left(\mathrm{Q}^{\mathrm{dup}}, \rho\right)$ is the directed 1-skeleton $\square_{d}$ of addimensional cube, and the increasing flip poset $\Gamma\left(\mathrm{Q}^{\mathrm{dup}}, \rho\right)$ is a boolean poset. Let $\phi: \square_{d} \rightarrow \Gamma\left(\mathrm{Q}^{\mathrm{dup}}, \rho\right)$ be the natural graph isomorphism which sends 0 to $\mathrm{P}\left(\mathrm{Q}^{\mathrm{dup}}, \rho\right)$ and 1 to $\mathrm{N}\left(\mathrm{Q}^{\mathrm{dup}}, \rho\right)$. It sends the edge labeling $\lambda$ of $\square_{d}$ (see Example 1.2 to the positive and negative edge labelings $\mathrm{p}$ and $\mathrm{n}$ of the subword complex $\mathcal{S C}\left(\mathrm{Q}^{\mathrm{dup}}, \rho\right)$. More precisely, $\lambda\left(\varepsilon \rightarrow \varepsilon^{\prime}\right)=\mathrm{p}\left(\phi(\varepsilon) \rightarrow \phi\left(\varepsilon^{\prime}\right)\right)=\mathrm{n}\left(\phi(\varepsilon) \rightarrow \phi\left(\varepsilon^{\prime}\right)\right)-1$. Thus, $\phi$ sends the $\lambda$-source tree of $\square_{d}$ to the source trees $\mathcal{P}\left(\mathrm{Q}^{\mathrm{dup}}, \rho\right)=\mathcal{N}^{*}\left(\mathrm{Q}^{\mathrm{dup}}, \rho\right)$, and the $\lambda$-sink tree of $\square_{d}$ to the sink trees $\mathcal{P}^{*}\left(\mathrm{Q}^{\mathrm{dup}}, \rho\right)=\mathcal{N}^{*}\left(\mathrm{Q}^{\mathrm{dup}}, \rho\right)$. See Example 1.2 and Figure 1 . Finally, the Möbius function on the increasing flip poset $\Gamma\left(\mathrm{Q}^{\mathrm{dup}}, \rho\right)$ is given by $\mu\left(\phi(\varepsilon), \phi\left(\varepsilon^{\prime}\right)\right)=(-1)^{\delta\left(\varepsilon, \varepsilon^{\prime}\right)}$ if $\varepsilon-\rightarrow \varepsilon^{\prime}$ (where $\delta$ denotes the Hamming distance on the vertices of the cube) and $\mu\left(\phi(\varepsilon), \phi\left(\varepsilon^{\prime}\right)\right)=0$ otherwise. See Example 1.4 


\section{References}

[Bjö80] Anders Björner. Shellable and Cohen-Macaulay partially ordered sets. Trans. Amer. Math. Soc., 260(1):159-183, 1980.

[BKPS06] Hervé Brönnimann, Lutz Kettner, Michel Pocchiola, and Jack Snoeyink. Counting and enumerating pointed pseudotriangulations with the greedy flip algorithm. SIAM J. Comput., 36(3):721-739 (electronic), 2006.

[BW96] Anders Björner and Michelle L. Wachs. Shellable nonpure complexes and posets. I. Trans. Amer. Math. Soc., 348(4):1299-1327, 1996.

[CLS13] Cesar Ceballos, Jean-Philippe Labbé, and Christian Stump. Subword complexes, cluster complexes, and generalized multi-associahedra. J. Algebraic Combin., 2013.

[Hum90] James E. Humphreys. Reflection groups and Coxeter groups, volume 29 of Cambridge Studies in Advanced Mathematics. Cambridge University Press, Cambridge, 1990.

[KM04] Allen Knutson and Ezra Miller. Subword complexes in Coxeter groups. Adv. Math., 184(1):161-176, 2004.

[KM05] Allen Knutson and Ezra Miller. Gröbner geometry of Schubert polynomials. Ann. of Math. (2), 161(3):1245-1318, 2005.

[KM12] Myrto Kallipoliti and Henri Mühle. On the EL-shellability of the cambrian semilattices. Preprint arXiv:1206.6248, 2012.

[PP12] Vincent Pilaud and Michel Pocchiola. Multitriangulations, pseudotriangulations and primitive sorting networks. Discrete Comput. Geom., 48(1):142-191, 2012.

[PS09] Vincent Pilaud and Francisco Santos. Multitriangulations as complexes of star polygons. Discrete Comput. Geom., 41(2):284-317, 2009.

[PS11] Vincent Pilaud and Christian Stump. Brick polytopes of spherical subword complexes: A new approach to generalized associahedra. Preprint, arXiv:1111.3349, 2011.

[PS12] Vincent Pilaud and Christian Stump. EL-labelings and greedy flip trees for subword complexes. Preprint, arXiv:1210.1435, 2012.

[PV96] Michel Pocchiola and Gert Vegter. Topologically sweeping visibility complexes via pseudotriangulations. Discrete Comput. Geom., 16(4):419-453, 1996.

[Rea06] Nathan Reading. Cambrian lattices. Adv. Math., 205(2):313-353, 2006.

[Rea07] Nathan Reading. Sortable elements and Cambrian lattices. Algebra Universalis, 56(3-4):411437, 2007.

[RSS08] Günter Rote, Francisco Santos, and Ileana Streinu. Pseudo-triangulations — a survey. In Surveys on discrete and computational geometry, volume 453 of Contemp. Math., pages $343-$ 410. Amer. Math. Soc., Providence, RI, 2008. 